\title{
Utilização da técnica do varal para angioplastia de estenose de veia central com stent-graft
}

\author{
Utilization of the through-and-through technique for central vein angioplasty using stent-graft \\ Ricardo Wagner da Costa Moreira', David Domingos Rosado Carrilho², Liana Berúcia Freire de Oliveira', \\ Charmy Cleython Fernandes de Araújo ${ }^{4}$, Raissa Gabriela Vieira da Câmara Barros ${ }^{5}$, Bruno Alexandre Barbosa do Nascimento ${ }^{5}$
}

\begin{abstract}
Resumo
A estenose de veia central é uma das situações mais frequentes em pacientes com insuficiência renal crônica em hemodiálise. A angioplastia com o uso de stent-graft tem obtido bons resultados nestes casos. O sistema de liberação dos stents é de calibre maior, podendo dificultar sua navegabilidade em áreas de estenose ou tortuosidade acentuadas. A técnica do varal é comumente utilizada para o tratamento endovascular do aneurisma de aorta, permitindo atingir bom mecanismo de estiramento e facilitando a navegação do sistema de entrega da endoprótese. Descrevemos o caso de uma angioplastia de veia central com stent-graft na qual foi utilizada a técnica do varal para permitir a transposição da área de estenose.
\end{abstract}

Palavras-chave: derivação arteriovenosa cirúrgica; angioplastia; diálise renal.

\begin{abstract}
The central vein stenosis is one of the most common conditions in patients with chronic kidney failure in a hemodialysis program. In these cases, angioplasty using stent-grafts has obtained good results. The stent-graft delivery system is generally of large diameter what can hinder its navigability in very severe stenosis or tortuosities. The through-and-through technique is commonly used for endovascular treatment of aortic aneurysms, making possible to achieve a good mechanism of stretch and easy navigation of the endograft delivery system. We report a case of a central venous stenosis in wich a stent-graft was inserted using the through-and-through technique in order to cross the lesion.
\end{abstract}

Keywords: arteriovenous shunt, surgical; angioplasty; renal dialysis.

\section{Introdução}

A estenose ou oclusão de veia central é um problema comum nos pacientes com insuficiência renal crônica e geralmente está relacionada ao uso prévio de cateteres ${ }^{1}$. A incidência do problema tem sido relatada na literatura como presente em 25 a $40 \%$ dos pacientes em hemodiálise ${ }^{2}$. Essas lesões comumente são muito rígidas e de difícil recanalização quando ocluídas, de forma que quando se tornam sintomáticas podem levar a edema do membro homolateral ao acesso arteriovenoso ou edema da face ${ }^{1}$. De acordo com a
Kidney Disease Outcomes Quality Initiative (KDOQI), toda fístula arteriovenosa (FAV) com estenose de pelo menos $50 \%$ deve ser tratada ${ }^{3}$. Opções terapêuticas disponíveis são: ligadura da fístula arteriovenosa, intervenção cirúrgica com criação de uma ponte veno-venosa ou abordagem endovascular com angioplastia transluminal percutânea da lesão estenótica com ou sem a colocação de stent ${ }^{1}$.

Apesar de não existirem estudos na literatura que indiquem a superioridade do stent em relação à angioplastia com balão, há claramente um grupo de pacientes que não é responsivo somente a este tipo de angioplastia, necessitando

Trabalho realizado no Hospital Promater - Natal (RN), Brasil.

Trabalho apresentado como pôster eletrônico durante o Congresso SoBRICE 2011, entre 10 e 13 de agosto de 2011 em Búzios (RJ).

${ }^{1}$ Cirurgião Vascular da Clínica Vascular de Natal; Professor auxiliar da Universidade Federal do Rio Grande do Norte (UFRN) - Natal (RN), Brasil.

${ }^{2}$ Cirurgião Vascular da Clínica Vascular de Natal - Natal (RN), Brasil.

${ }^{3}$ Cirurgiã Vascular da Clínica Vascular de Natal; Cirurgiã Vascular da UFRN - Natal (RN), Brasil.

${ }^{4}$ Médico Nefrologista do Hospital Estadual Monsenhor Walfredo Gurgel e do Natal Hospital Center - Natal (RN), Brasil.

${ }^{5}$ Acadêmica(o) de Medicina da UFRN - Natal (RN), Brasil.

Fonte de financiamento: nenhuma.

Conflito de interesses: nada a declarar.

Submetido em: 05.12.11. Aceito em: 02.03.12.

J Vasc Bras. 2012;11(3):246-249. 
do implante de stent $t^{4,5}$. O uso de stents recobertos, ou stent-graft, parece trazer melhores resultados do que quando utilizados os não recobertos, com perviedade relatada de até $100 \%$ em 9 meses ${ }^{4,5}$.

$\mathrm{O}$ uso de stent-graft em veias centrais requer sempre a utilização de calibres maiores devido ao diâmetro das veias subclávias e braquiocefálicas. O cateter sobre o qual os stent-grafts de grande calibre estão montados (shaft) apresenta diâmetro também maior, o que pode tornar mais difícil a navegação do sistema em áreas tortuosas ou a transposição em áreas de estenose muito acentuada, condições frequentemente encontradas em pacientes com estenose de veia central.

$\mathrm{O}$ tratamento endovascular de aneurisma da aorta abdominal e torácica utiliza endopróteses de grande calibre do sistema de entrega, e, em aortas tortuosas ou com áreas de estreitamento, a navegação do sistema pode tornar-se difícil. Nessas condições, algumas técnicas mostram-se úteis, como é o caso da do varal ${ }^{6,7}$.

A técnica do varal utilizada para correção de aneurisma de aorta consiste na passagem de um fio-guia da artéria femoral até a braquial e posterior tração das suas extremidades, com retificação por um mecanismo de estiramento. Dessa forma, proporciona melhor passagem do sistema condutor da endoprótese e posterior colocação do stent com maior facilidade ${ }^{4,8,9}$.

O presente artigo tem como objetivo relatar o procedimento de angioplastia de veia braquiocefálica utilizando stent-graft associado à técnica do varal como forma de contornar a dificuldade de progressão do sistema de liberação, permitindo a realização do procedimento endovascular com sucesso.
O Comitê de Ética em Pesquisa do Hospital Universitário Onofre Lopes, da Universidade Federal do Rio Grande do Norte, determina que, para os artigos no formato de relato de caso, é necessário apenas o consentimento informado por escrito do paciente, o qual foi feito neste caso.

\section{Relato do caso}

Paciente do sexo masculino, com 57 anos, hipertenso e diabético, em hemodiálise por meio de uma fístula arteriovenosa bráquio-basílica esquerda. Tem história de uso de múltiplos cateteres para hemodiálise de longa e curta permanência em região cervical e femoral bilateralmente. A aproximadamente três meses da realização do estudo, referiu que houve início de edema progressivo no membro superior esquerdo e face, associado a pequenas variações de fluxo durante as sessões de hemodiálise, porém nunca inferior a $350 \mathrm{~mL} / \mathrm{min}$. No exame físico apresentava edema acentuado em todo o membro superior esquerdo e hemiface esquerda, além de FAV com bom frêmito.

O paciente foi submetido a arteriografia, a qual evidenciou FAV bráquio-basílica pérvia, anastomose sem estenoses e presença de estenose significativa em toda a extensão da veia braquiocefálica esquerda, com circulação colateral a nível cervical (Figura 1).

Em um segundo momento foi programado o tratamento endovascular da estenose encontrada, utilizando um stent-graft, realizada punção da veia basílica e colocado introdutor $4 \mathrm{~F}$ para realizar angiografias de controle durante o procedimento. Na sequência houve punção da veia femoral comum direita e colocação de introdutor 12F. Seguiu-se
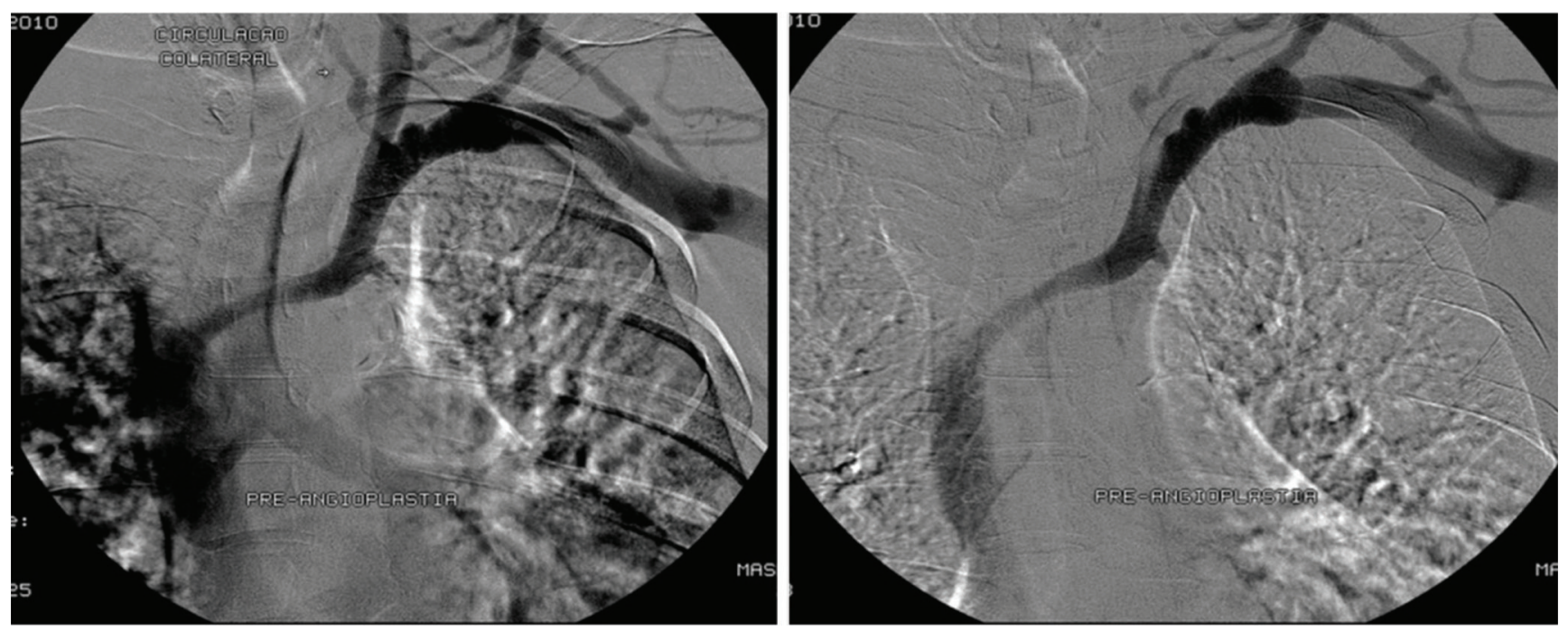

Figura 1. Estenose longa envolvendo toda a veia braquiocefálica esquerda com presença de circulação colateral cervical. 

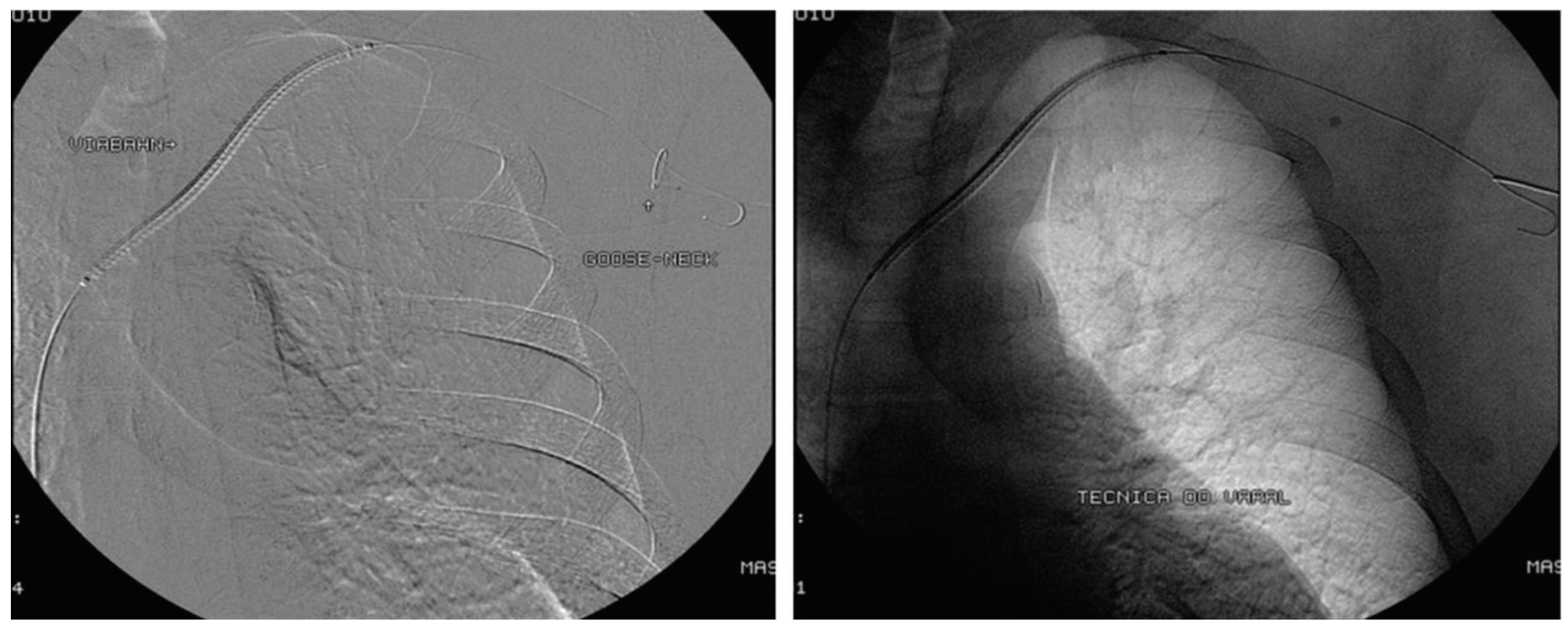

Figura 2. Estabelecimento da técnica do varal utilizando cateter tipo goose-neck inserido por introdutor posicionado na veia basílica.

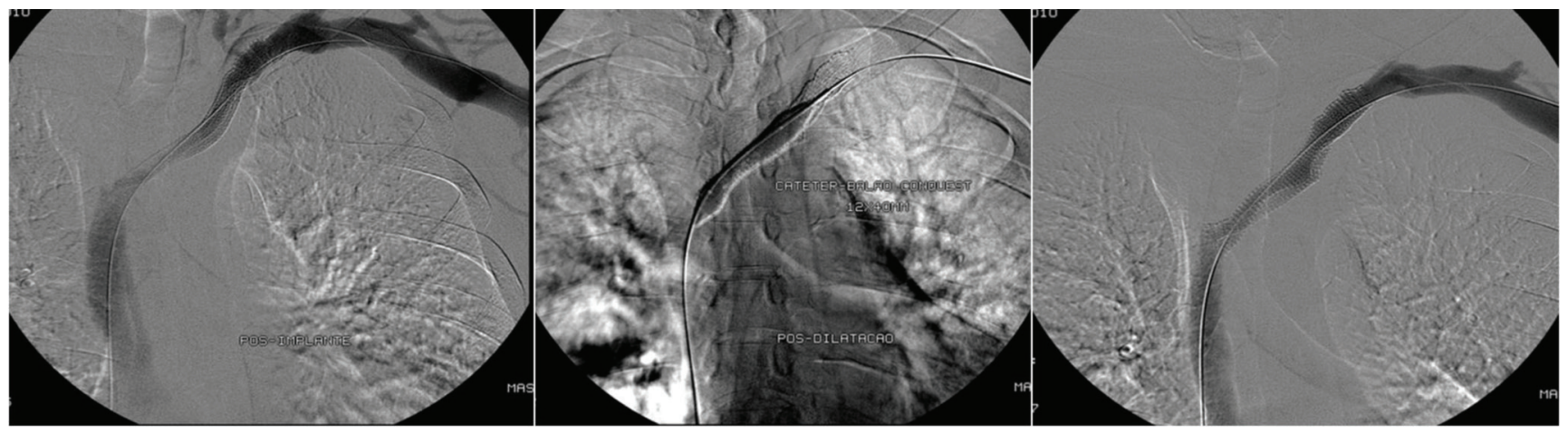

Figura 3. Liberação do stent-graft, pós-dilatação com cateter-balão e aspecto final com detalhe para o desaparecimento da circulação colateral.

heparinização sistêmica com 5 mil UI de heparina. Houve transposição da área de estenose utilizando fio-guia 0,035" hidrofílico e cateter Headhunter 5F, e troca do fio-guia 0,035” por um fio-guia 0,025 " adequado ao stent-graft. Foi utilizado um stent-graft Viabahn ${ }^{\circledR} 13 \times 100$ mm (W. L. Gore, Flagstaff, EUA), mas não se conseguiu a transposição da lesão com o sistema de entrega do Viabahn, e qualquer tentativa de empurrar o sistema com mais força ocasionava a saída do fio-guia da área de estenose, sendo necessário transpor a estenose novamente.

Por meio do introdutor posicionado na veia basílica foi introduzido um cateter goose-neck, e a extremidade do fio-guia 0,025 " posicionada na veia axilar foi "laçada", estabelecendo-se, assim, a técnica do varal e proporcionando um mecanismo de estiramento suficiente para permitir a navegação do sistema (Figura 2). Após isto, o Viabahn conseguiu transpor a lesão e foi adequadamente posicionado e liberado. Foi realizada pós-dilatação com cateter-balão Conquest $^{\circledR} 12 \times 40 \mathrm{~mm}$ (Bard, Covington, EUA). Para o posicionamento adequado deste balão foi necessário manter a técnica do varal, porém utilizando um fio-guia 0,035 ” Amplatz Extra-stiff (Figura 3). Procedeu-se à retirada dos introdutores e hemostasia por compressão local.

A angiografia de controle mostrou tratamento adequado da lesão e desaparecimento da circulação colateral, um sinal indireto da efetividade da angioplastia.

\section{Discussão}

As estenoses e tortuosidades acentuadas podem tornar extremamente difícil a progressão dos cateteres-balão ou dispositivo de liberação dos stents-graft, por estes serem mais calibrosos. Neste caso, a dificuldade ocorreu devido ao calibre do sistema de entrega do stent-graft dificultar a 
passagem do sistema na área de estenose e, principalmente, à dificuldade de navegabilidade nas tortuosidades presentes ao longo da veia subclávia e braquiocefálica.

A chamada técnica do varal é classicamente descrita para a correção endovascular de aneurisma de aorta abdominal e torácica ${ }^{2,6,7}$. Conseguimos transportar a experiência adquirida com o uso desta técnica para solucionar o problema encontrado durante uma angioplastia de veia central. Neste caso, o fio-guia foi passado desde a veia femoral até a da FAV, proporcionando o mecanismo de estiramento suficiente para conseguir a navegabilidade do sistema de liberação do stent-graft.

Dessa forma, a técnica do varal mostra que é útil não apenas para os casos de tratamento endovascular de aneurisma da aorta, mas também obtendo sucesso em casos de angioplastia de veia central.

\section{Referências}

1. Lucas C, Gil C, Martinho A, Pais MJ. Terapêutica endovascular de estenoses venosas centrais: uma avaliação prospectiva. Rev Port Nefrol Hipert. 2006;20(2):117-23.

2. Lumsden AB, MacDonald MJ, Isiklar H, et al. Central venous stenosis in the hemodialysis patient: incidence and efficacy of endovascular treatment. Cardiovasc Surg. 1997;5(5):504-9. PMid:9464608.

3. National Kidney Foundation [Internet]. The National Kidney Foundation Kidney Disease Outcomes Quality Initiative (NKF DOQI). [updated 2006 Jan 01; cited 2011 Dec 05]. Available from: http://www.kidney.org/professionals/kdoqi/

4. Kundu S. Central venous disease in hemodialysis patient: prevalence, etiology and treatment. J Vasc Access. 2010;11(1):1-7. PMid:20119911.
5. Jones RG, Willis AP, Jones C, MCCafferty IJ, Riley PL. Long-term results of stent-graft placement to treat central venous stenosis and occlusion in hemodialysis patients with arteriovenous fistulas. J Vasc Interv Radiol. 2011;22(9):1240-5. PMid:21764328.

6. Carnevale FC, Nasser F, Oliveira C, Borges MV, Affonso BB. Aneurismas de aorta: até onde expandir a indicação do tratamento endovascular? Rev Bras Cardiol Invas. 2006;14(1):82-8.

7. Yang J, Zuo J, Yang L, et al. Endovascular stent-graft treatment of thoracic aortic dissection. Interact Cardiovasc Thorac Surg. 2006;5(6):68891. http://dx.doi.org/10.1510/icvts.2006.135442

8. IngrundJC, Nasser F, Jesus-Silva SG, et al. Tratamento híbrido das doenças complexas da aorta torácica. Rev Bras Cir Cardiovasc. 2010;25(3):303-10. http://dx.doi.org/10.1590/S0102-76382010000300005

9. Neves AAG, Oliveira AGNM, Beck RT, Santos RV, Moreira FCP, Amato ACM. Tratamento endovascular de pseudoaneurisma de aorta torácica com fístula aorto-brônquica em pós-operatório tardio de cirurgia de correção de coarctação de aorta. J Vasc Bras. 2011;10(1):64-7. http://dx.doi.org/10.1590/ S1677-54492011000100012

Correspondência Ricardo Wagner da Costa Moreira Av. Campos Sales, 759 / apto 800 - Tirol CEP 59020-300 - Natal (RN), Brasil E-mail: ricardowcm@yahoo.com.br

Contribuições dos autores Concepção e desenho do estudo: RWCM Análise e interpretação dos dados: RWCM, DDRC, LBFO Coleta de dados: RGVCB, BABN Redação do artigo: RWCM, RGVCB, BABN Revisão crítica do texto: DDRC, LBFO, CCFA Aprovação final do artigo*: RWCM, DDRC, LBFO, CCFA, RGVCB, BABN Análise estatística: RWCM Responsabilidade geral pelo estudo: RWCM *Todos os autores leram e aprovaram a versão final submetida ao J Vasc Bras 